\title{
ASPECTOS COMPARATIVOS DAS COMUNIDADES DE ARANHAS (ARANEAE) EM DOIS REMANESCENTES DE MATA ATLÂNTICA DO ESTADO DA BAHIA, BRASIL
}

\author{
Kátia Regina Benati, João Pedro Souza-Alves, Elizabete Alves Silva, Marcelo Cesar Lima Peres e \\ Érica Oliveira Coutinho
}

Biota Neotropica v5 (n1a) - http://www.biotaneotropica.org.br/v5n1a/pt/abstract?article+BN005051a2005

Recebido em 07/12/2003

Aceito em 01/02/2005

Universidade Católica do Salvador, Instituto de Ciências Biológicas, Centro de Ecologia e Conservação Animal (ECOA), Av. Prof ${ }^{\circ}$ Pinto de Aguiar, 2589, Pituaçu, 41740-090, Salvador, Bahia, Brasil. E-mail: katiabenati@yahoo.com.br

\begin{abstract}
The Atlantic Rainforest has suffered a rapid fragmentation process which has directly affected its biodiversity. The aim of the present study was to inventory of the spider community composition in two Atlantic Rainforest remnants in the state of Bahia, in order to enable a comparison of the abundance, richness and species diversity between the plots. The aim is to contribute towards further studies on the dynamics of the forest remnants. The sampled areas were of distinctive sizes, the Pituaçu Metropolitan Park (PMP) in Salvador with 425 hectares and the Matinha City Park (PMM) in Itapetinga with nine hectares. The spiders were collected using the nocturnal manual collection method in a 30m x $5 \mathrm{~m}$ transect for an hour, totalling 10 hours of sampling effort. A total of 362 individuals were collected, of which 152 were, belonging to 20 families, and 60 species. Of these, 37 species of 12 families were collected in the PMP and 30 species of 16 families in the PMM. The abundance of the most frequent families (minimum of ten captures) did not differ between the plots ( $U=46,00$; $p=0.7959$ ), with a Sorensen similarity index of 82,4\%. Also, the estimates of the abundance and richness do not differ between the areas ( $U=65.000 ; p=0.2799$ and $U=20755, p=0.1451$, respectively). Nevertheless, the diversity was higher in the PMP $(D=8.1922)$ than the PMM ( $\mathrm{D}=6.8032)$. The results suggest that the similarities between the areas may be a consequence of factors related to the fragmentation processes, such as disturbance history. However, other factors, such as the shape of the remnants and the type of neighborhood, apart from other variables, eg. the flora composition, may also interfere on the dynamics of the spider's communities in these fragments.
\end{abstract}

Key words: Atlantic Forest, Fragmentation, Biodiversity, Spiders, Bahia.

\section{Resumo}

A Mata Atlântica vem sofrendo um acelerado processo de fragmentação, o que está afetando sua biodiversidade. O objetivo do trabalho foi inventariar as comunidades de aranhas em dois remanescentes de Mata Atlântica do Estado da Bahia, permitindo uma comparação entre a abundância, riqueza e diversidade de espécies entre estas áreas, visando fornecer subsídios para estudos da dinâmica de fragmentos florestais. Foram amostrados remanescentes com tamanhos bastante distintos, o Parque Metropolitano de Pituaçu - Salvador (PMP) com 425 ha e o Parque Municipal da Matinha Itapetinga (PMM) com nove ha. As aranhas foram capturadas por coleta manual noturna, num transecto de trinta por cinco metros durante uma hora, com esforço total de 10h. Foi coletado um total de 362 indivíduos, distribuídos em 20 famílias, com 152 adultos e 60 espécies, sendo 37 spp. e 12 famílias no PMP e 30 spp. e 16 famílias no PMM. Ao comparar a abundância das famílias mais freqüentes (mínimo de dez capturas) não houve diferenças significativas entre as áreas (U=46,00; p=0,7959), com um índice de similaridade de Sorensen de 82,4\%. Quando comparada a riqueza estimada e a abundância de espécies, não houve diferenças significativas entre as duas áreas ( $U=65.000 ; p=0,2799)$ e ( $U=20755, p=0,1451)$, respectivamente. Já a diversidade encontrada nas duas áreas, foi maior no PMP $(\mathrm{D}=8,1922)$ do que no PMM $(\mathrm{D}=6,8032)$. A partir dos resultados, sugere-se que as semelhanças encontradas entre as áreas podem estar relacionadas a fatores ligados aos processos da fragmentação, como histórico de perturbações. Contudo, sugerimos que seja feita uma avaliação a posteriori de outros fatores, como a forma do fragmento, o tipo de vizinhança e efeito de borda, além de outras variáveis, i.e. da composição florística, que possam estar interferindo na dinâmica destes fragmentos e influenciando na comunidade destas aranhas. Palavras-chave: Mata Atlântica, Fragmentação, Biodiversidade, Aranhas, Bahia. 


\section{Introdução}

As florestas tropicais são conhecidas por sua alta biodiversidade (Briggs 1996, Burslem et al. 2001), são os biomas mais ricos e diversos, apresentando uma complexidade estrutural que favorece a existência de muitos nichos ecológicos (Mantovani 2003), porém, são pouco estudadas (Myers 1997) e sujeitas a várias ações naturais e não naturais (DeSouza et al. 2001). Inúmeras espécies diminuíram rapidamente sua abundância, e algumas até foram extintas em conseqüência da caça predatória, destruição de habitats e ação de novos predadores e competidores (Primack \& Rodrigues 2002).

No Brasil, a devastação de áreas florestais vem atingindo proporções bastante significativas. Na Mata Atlântica, a maior parte dos remanescentes florestais encontra-se na forma de pequenos fragmentos (Viana 1995). Este bioma é considerado uma área prioritária para conservação biológica do planeta (Dário \& Almeida 2000) devido ao seu nível de ameaça, taxa de endemismo e diversidade da fauna e flora, estando em quarto lugar dentre os 25 hotspots mundiais (Myers et al. 2000).

Os remanescentes florestais constituem hoje um dos maiores desafios para a conservação biológica (EspíritoSanto et al. 2002), pois ao se diminuir uma área florestal, pode-se reduzir significativamente o número de espécies, afetar a dinâmica das populações de animais e vegetais, além de comprometer a regeneração natural das florestas (Harris 1974), tornando-as mais susceptíveis a modificações ambientais ou a variações demográficas (Metzger 2003). O número de espécies que um fragmento pode suportar, e suas respectivas taxas de extinção, dependem do seu tamanho, estrutura do habitat e povoamento (MacArthur \& Whitmore 1979), além do grau de isolamento, tipo de vizinhança, histórico de perturbações (Viana \& Pinheiro 1998) e conectividade (Metzger 2000). Estes remanescentes são ainda pouco conhecidas, pois os inventários, mesmo que rápidos, são quase inexistentes para várias regiões (Vieira 2003), exigindo o desenvolvimento de estratégias para conservação, bem como a recuperação de áreas degradadas (Conroy \& Noon 1996).

Os efeitos da fragmentação vêm sendo usualmente analisados a partir de estudos de ecologia de comunidades em remanescentes com diferentes características espaciais (Kelt 2000, Metzger 2000, Collinge \& Palmer 2002, SteffanDewenter 2003), podendo ser avaliados em nível biótico por meio de mudanças na estrutura e dinâmica das comunidades vegetais, na diversidade e abundância da fauna e nas interações entre ambas (Murcia 1995) - e abiótico, que são efeitos mais facilmente perceptíveis e quantificáveis, como o aumento da temperatura, redução da umidade relativa do ar e retenção da precipitação (Kapos 1989). Contudo, esses estudos ocorrem em diferentes áreas ou têm curta duração. Para avaliar o impacto dessas fragmentações, o primeiro passo é inventariar a fauna e a flora de uma determinada área, obter um conhecimento mínimo sobre os organismos ocorrentes no local e, a partir daí, desenvolver projetos de conservação ou preservação (Pearson 1994).

É muito difícil, se não impossível, determinar a riqueza total de espécies de uma determinada área, por menor que seja este ambiente, pois existe uma alta diversidade de organismos (May 1988). A seleção de organismos indicadores de diversidade deve levar em conta as características biológicas e ecológicas do grupo, o conhecimento disponível sobre a sistemática e as dificuldades de coleta (Lawton et al. 1998). Desta forma, estudos sobre a biodiversidade de táxons megadiversos, como os artrópodes, podem contribuir muito com o avanço, tanto do conhecimento básico sobre o funcionamento dos ecossistemas, quanto para monitoramento e planejamento de programas de conservação e uso sustentado (Kremen et al. 1993). O conhecimento sobre os invertebrados de Mata Atlântica é bastante escasso, tornando-se necessário um esforço conjunto para inventariar áreas remanescentes, avaliar a possibilidade de proteção e manejo, além de fundamentar a conservação efetiva daquelas de maior valor biológico (Conservation International do Brasil et al. 2000).

As aranhas (Arachnida: Araneae), apesar de ser um grupo pouco estudado (Coddington \& Levi 1991), são consideradas de grande importância ecológica (Simó et al. 1994). Compreendem uma porção bastante significativa da diversidade de artrópodes terrestres (Toti et al. 2000), constituindo um dos grupos mais diversos e abundantes, com 38.663 espécies descritas até o momento (Platnick 2004), amplamente distribuídas em todas as regiões do mundo, exceto na Antártida (Platnick 1995). A maioria das espécies é sensível a diversos fatores físicos, como temperatura, umidade, vento e intensidade luminosa, e biológicos, como estrutura da vegetação e disponibilidade de alimento (Wise 1993, Foelix 1996). Alguns autores sugerem que a estrutura do ambiente pode influenciar na composição e riqueza das comunidades de aranhas (Rushton et al. 1987, Foelix 1996, Santos 1999). Se considerarmos a grande riqueza em espécies, a facilidade de amostragem e a sensibilidade a diversos fatores ambientais, as aranhas são consideradas como espécies guarda-chuva (Lambeck 1997), i.e., apresentam mais exigências do que outros grupos que vivem no mesmo habitat (Metzger 2003), sendo indicadas para avaliar as diferenças ambientais entre diversos meios.

Este trabalho teve como objetivo inventariar as comunidades de aranhas em dois remanescentes de Mata Atlântica do Estado da Bahia, permitindo uma comparação entre a abundância, riqueza e diversidade de espécies entre estas áreas, visando fornecer subsídios para estudos da dinâmica de fragmentos florestais. 


\section{Metodologia}

\section{1. Áreas de estudo}

O trabalho foi realizado em dois remanescentes de Mata Atlântica do Estado da Bahia. O Parque Metropolitano de Pituaçu - PMP (12 58'076" S e 38 24'746” W), localizado na área urbana do município de Salvador, possui uma extensão de 425 ha, com áreas de mata secundária, mata ciliar e restinga; possuindo uma ampla área de transição entre essas fisionomias, em estágio inicial, médio e avançado de regeneração (Conceição et al. 1998); e o Parque Municipal da Matinha - PMM (15 $14^{\circ}$ '407' S e 40 14 '117' W), situando na região urbana do município de Itapetinga, no sudoeste do Estado, possuindo uma extensão total de 40 ha, porém com apenas nove ha de mata. Ambas as áreas sofrem impactos do trânsito regular de moradores do entorno e público visitante.

\subsection{Amostragem}

As áreas foram escolhidas aleatoriamente, onde foram definidos 10 pontos de coleta em ambiente de borda, obtendo um total de 10 horas amostrais em cada fragmento. $\mathrm{O}$ método de amostragem aplicado foi a coleta manual noturna (look down e look up), constituída de um transecto de trinta por cinco metros, sendo as coletas realizadas por dois coletores, com um esforço de uma hora. No momento das coletas, as aranhas foram acondicionadas em álcool $(70 \%)$ e encaminhadas para identificação no Centro de Ecologia e Conservação Animal - ECOA / Instituto de Ciências Biológicas - ICB / Universidade Católica do Salvador - UCSal, onde os espécimes foram depositados.

\subsection{Análise dos dados}

As análises estatísticas foram feitas usando-se o programa Bioestat 2.0 (Ayres et al. 2000). Para comparar a abundância de famílias, riqueza em espécies e abundância de espécies entre as áreas foi utilizado o teste Mann-Whitney. As estimativas de riqueza foram obtidas no EstimateS 5.0 (Colwell 1997) e o índice de diversidade através do Diversity ${ }^{\odot}$ 2.2 (Henderson \& Seaby 1998). O índice de similaridade de Sorensen foi utilizado para avaliar o grau de semelhança da composição das famílias mais abundantes entre as áreas.

\section{Resultados e Discussão}

Foi coletado um total de 362 indivíduos, distribuídos em 20 famílias. No PMP foram registrados 159 espécimes e 11 famílias, e no PMM 203 e 18 (Figura 1). As áreas não apresentam diferença na abundância das famílias mais freqüentes (mínimo de dez capturas), que representaram $90,33 \%$ do total de indivíduos ( $\mathrm{U}=46,00 ; \mathrm{p}=0,7959)$, e apresentam um alto índice de similaridade de Sorensen (82,4\%). Dentre estas famílias, quatro representaram 63,6\% das aranhas amostradas, Pholcidae (18,3\%), Araneidae (16,8\%), Salticidae $(14,7 \%)$ e Theridiidae $(13,8 \%)$, observando-se um elevado número de aranhas construtoras de teia $(48,9 \%)$. Ao avaliarmos as duas áreas separadamente, o PMP teve predominância de Salticidae (25,7\%), Pholcidae (17,8\%) e Araneidae (15,8\%), já o PMM Theridiidae (20,0\%), Pholcidae (18,9\%) e Araneidae (17,7\%).

Considerando-se o fato das amostras terem sido realizadas em ambiente de borda e que os fragmentos têm histórico de intensas perturbações antrópicas, a predominância de aranhas construtoras de teias em ambas as

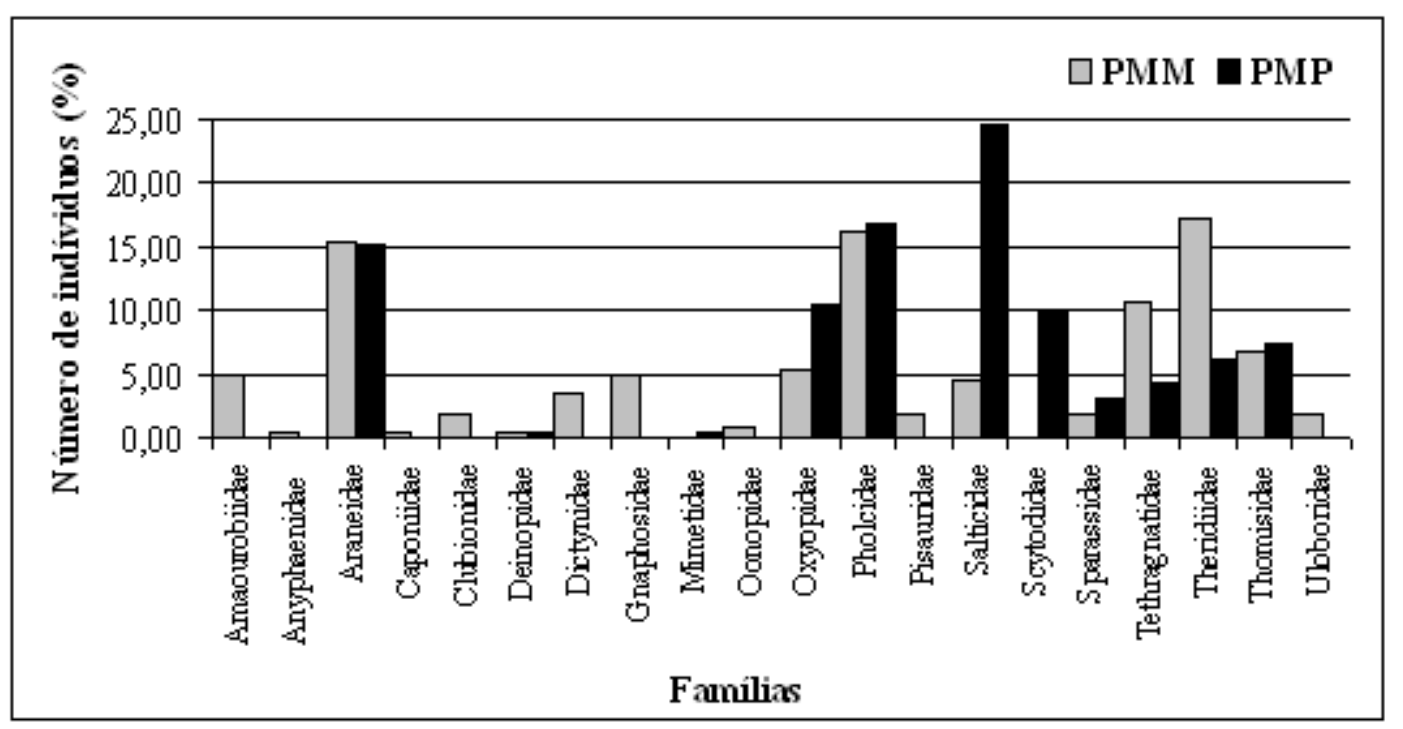

Figura 1: Freqüencia das famílias encontradas nas áreas de estudo. PMP = Parque Metropolitano de Pituaçu (Salvador - Bahia) e PMM = Parque Municipal da Matinha (Itapetinga - Bahia). 
áreas sugere que algumas espécies destas famílias podem estar bem adaptadas a viver em ambientes abertos, como bordas de mata ou áreas perturbadas, como foi demonstrado por Santos (1999) para algumas espécies de Araneidae e Theridiidae. Resultados semelhantes foram encontrados por outros autores, como Whitmore et al (2002), que encontraram uma elevada freqüência de espécies da família Araneidae (31 spp.) representando cerca de $12 \%$ das espécies coletadas em ambiente de Savana na África, e Peres (2001), que verificou duas espécies de Araneidae (Alpaida antonio e Alpaida sp.) exibindo preferência por ambiente de clareira natural em um remanescente de Mata Atlântica inserida na cidade de Recife (Pernambuco - Brasil). Desta forma, embora os ambientes de borda geralmente apresentem menor complexidade estrutural, o que possivelmente reduz a oferta de substrato adequado para a fixação de suas teias, as espécies coletadas, pelo menos as mais freqüentes (Metazygia gregalis e Eriophora sp.), provavelmente adaptaram-se às alterações que ocorrem na borda, buscando substratos alternativos para a fixação das teias. A elevada ocorrência de Pholcidae em áreas de borda não era esperada, visto que, segundo Santos (1999) alguns gêneros desta família, como Priscula, Mesabolivar e Metagonia exibem preferência por ambientes úmidos e sombreados. No entanto, nossos resultados demonstram que algumas espécies dos gêneros Priscula e Mesabolivar podem ser tolerantes a ambientes de borda, que possuem menor umidade e sombreamento. Peres (2001) verificou que embora a espécie Priscula sp. exibisse preferências por ambientes de

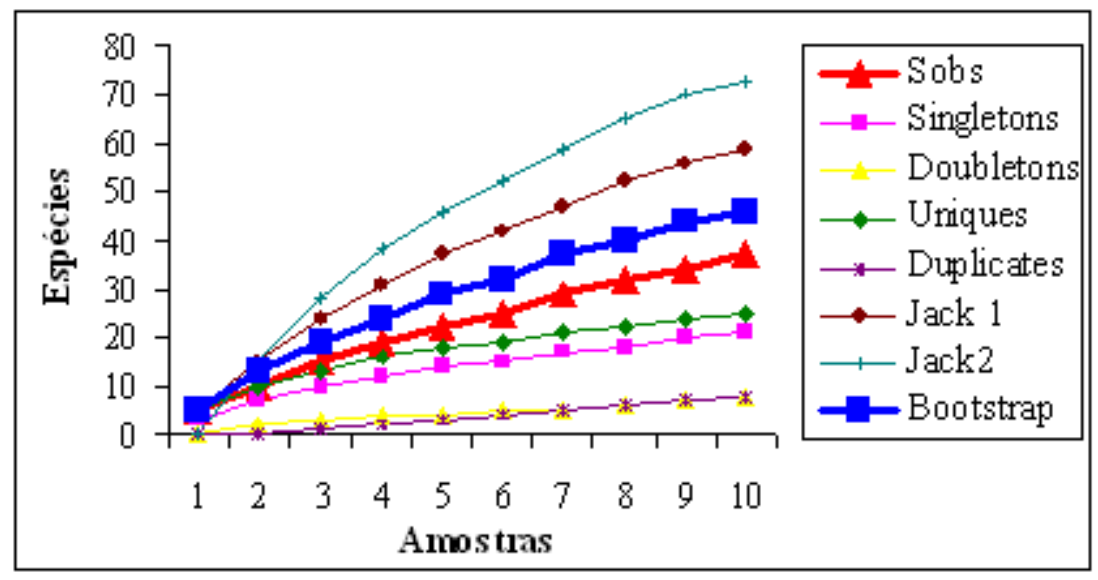

Figura 2: Estimativas de riqueza de espécies no Parque Municipal da Matinha (Itapetinga - Bahia). Sobs = espécies observadas.

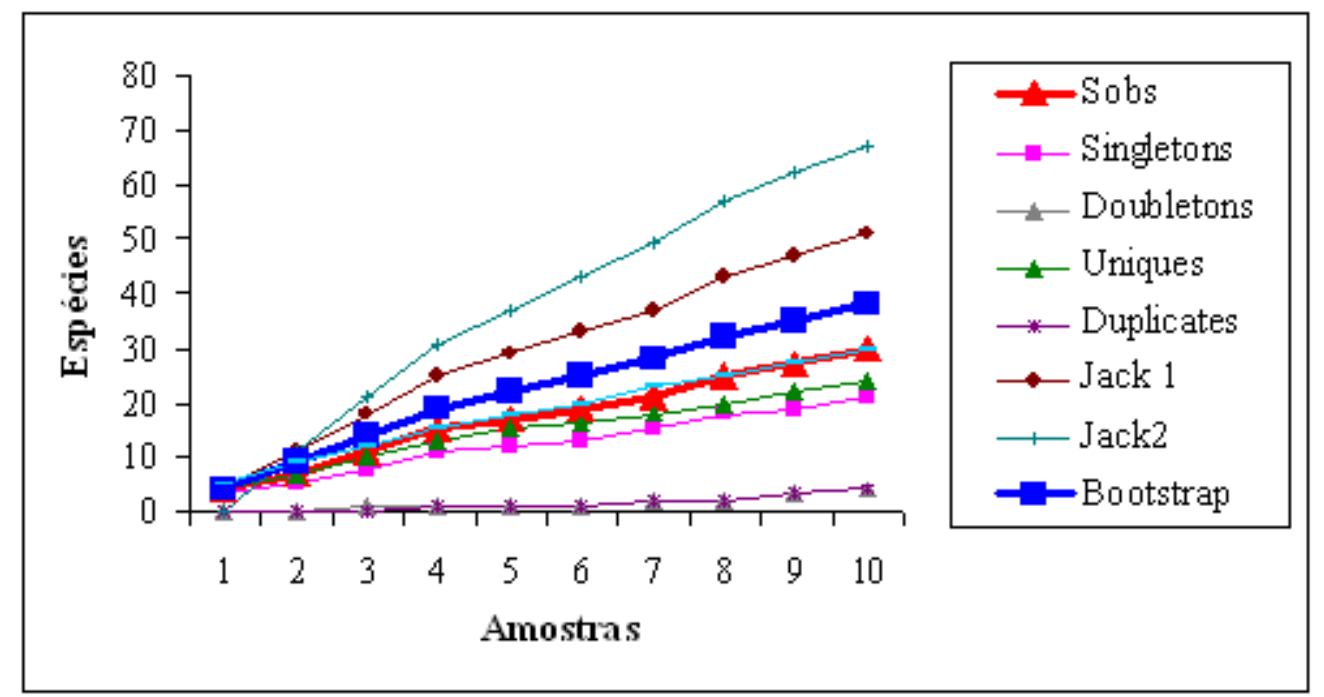

Figura 3: Estimativas de riqueza de espécies no Parque Metropolitano de Pituaçu (Salvador - Bahia). Sobs = espécies observadas.

http://www.biotaneotropica.org.br 
Benati, K.R. (et al.) - Biota Neotropica, v5 (n1a) - BN005051a2005

\begin{tabular}{|c|c|c|c|c|c|}
\hline Família & Morfoespécie & PMP & PMM & Total & $\%$ \\
\hline Amaurobiidae & Amaurobiidae sp. & 0 & 2 & 2 & 1,32 \\
\hline \multirow[t]{3}{*}{ Anyphaenidae } & Anyphaenidae sp. & 2 & 0 & 2 & 1,32 \\
\hline & Wulfila sp. & 1 & 0 & 1 & 0,66 \\
\hline & Xiruana sp. & 0 & 1 & 1 & 0,66 \\
\hline \multirow[t]{11}{*}{ Araneidae } & Araneus tijuca & 3 & 0 & 3 & 1,97 \\
\hline & Argiope argentata & 2 & 1 & 3 & 1,97 \\
\hline & Cyclosa sp. & 2 & 2 & 4 & 2,63 \\
\hline & Eriophora sp. & 6 & 1 & 7 & 4,61 \\
\hline & Eustala sp. & 0 & 1 & 1 & 0,66 \\
\hline & Metazygia gregalis & 0 & 7 & 7 & 4,61 \\
\hline & Araneidae sp.1 & 1 & 0 & 1 & 0,66 \\
\hline & Araneidae sp.2 & 1 & 0 & 1 & 0,66 \\
\hline & Araneidae sp.3 & 7 & 0 & 7 & 4,61 \\
\hline & Araneidae sp.4 & 2 & 0 & 2 & 1,32 \\
\hline & Araneidae sp.5 & 2 & 0 & 2 & 1,32 \\
\hline Caponiidae & Caponiidae sp. & 0 & 1 & 1 & 0,66 \\
\hline Clubionidae & Elaver sp. & 1 & 0 & 1 & 0,66 \\
\hline Ctenidae & Nothroctenus sp. & 0 & 4 & 4 & 2,63 \\
\hline Deinopidae & Deinopis sp. & 1 & 0 & 1 & 0,66 \\
\hline Dictynidae & Dictyna sp. & 0 & 3 & 3 & 1,97 \\
\hline \multirow[t]{2}{*}{ Gnaphosidae } & Gnaphosidae sp.1 & 0 & 1 & 1 & 0,66 \\
\hline & Gnaphosidae sp.2 & 0 & 1 & 1 & 0,66 \\
\hline Mimetidae & Gelanor sp. & 1 & 0 & 1 & 0,66 \\
\hline \multirow[t]{2}{*}{ Oxyopidae } & Peucetia rubrolineata & 3 & 5 & 8 & 5,26 \\
\hline & Peucetia sp. & 1 & 1 & 2 & 1,32 \\
\hline \multirow[t]{2}{*}{ Pholcidae } & Blechroscelis sp. & 13 & 0 & 13 & 8,55 \\
\hline & Mesabolivar sp. & 0 & 23 & 23 & 15,13 \\
\hline \multirow[t]{9}{*}{ Salticidae } & Lyssomanis sp. & 1 & 1 & 2 & 1,32 \\
\hline & Thiodina sp. & 1 & 0 & 1 & 0,66 \\
\hline & Salticidae sp.1 & 3 & 0 & 3 & 1,97 \\
\hline & Salticidae sp.2 & 1 & 0 & 1 & 0,66 \\
\hline & Salticidae sp.3 & 0 & 1 & 1 & 0,66 \\
\hline & Salticidae sp.4 & 5 & 0 & 5 & 3,29 \\
\hline & Salticidae sp.5 & 1 & 0 & 1 & 0,66 \\
\hline & Salticidae sp. 6 & 1 & 0 & 1 & 0,66 \\
\hline & Salticidae sp.7 & 1 & 0 & 1 & 0,66 \\
\hline \multirow[t]{3}{*}{ Scytodidae } & Scytodes itapevi & 0 & 1 & 1 & 0,66 \\
\hline & Scytodidae sp.1 & 4 & 0 & 4 & 2,63 \\
\hline & Scytodidae sp.2 & 2 & 0 & 2 & 1,32 \\
\hline Sparassidae & Olios sp. & 0 & 1 & 1 & 0,66 \\
\hline \multirow[t]{5}{*}{ Tetragnathidae } & Leucauge aff. argira & 2 & 0 & 2 & 1,32 \\
\hline & Nephila clavipes & 1 & 0 & 1 & 0,66 \\
\hline & Tetragnathidae sp.1 & 1 & 0 & 1 & 0,66 \\
\hline & Tetragnathidae sp.2 & 0 & 1 & 1 & 0,66 \\
\hline & Tetragnathidae sp.3 & 1 & 0 & 1 & 0,66 \\
\hline \multirow[t]{7}{*}{ Theridiidae } & Episinus sp. & 1 & 0 & 1 & 0,66 \\
\hline & Tidarren sp. & 1 & 0 & 1 & 0,66 \\
\hline & Theridiidae sp.1 & 0 & 1 & 1 & 0,66 \\
\hline & Theridiidae sp.2 & 1 & 0 & 1 & 0,66 \\
\hline & Theridiidae sp.3 & 1 & 0 & 1 & 0,66 \\
\hline & Theridiidae sp.4 & 0 & 1 & 1 & 0,66 \\
\hline & Theridiidae sp.5 & 0 & 1 & 1 & 0,66 \\
\hline \multirow[t]{4}{*}{ Thomisidae } & Tmarus sp.1 & 0 & 1 & 1 & 0,66 \\
\hline & Tmarus sp.2 & 2 & 1 & 3 & 1,97 \\
\hline & Thomisidae sp.1 & 0 & 1 & 1 & 0,66 \\
\hline & Thomisidae sp.2 & 0 & 1 & 1 & 0,66 \\
\hline \multirow[t]{4}{*}{ Uloboridae } & Uloborus sp. & 0 & 2 & 2 & 1,32 \\
\hline & Uloboridae sp.1 & 1 & 0 & 1 & 0,66 \\
\hline & Uloboridae sp.2 & 0 & 1 & 1 & 0,66 \\
\hline & Uloboridae sp.3 & 0 & 2 & 2 & 1,32 \\
\hline Total & & 81 & 71 & 152 & 100,00 \\
\hline
\end{tabular}

Tabela 1: Lista de espécies das áreas amostradas. PMP = Parque Metropolitano de Pituaçu (Salvador - Bahia) e PMM = Parque Municipal da Matinha (Itapetinga - Bahia).

http://www.biotaneotropica.org.br 
mata, a mesma também foi bastante abundante em ambiente de clareira natural, que se caracterizam por temperatura mais elevadas, pouca umidade e intensa luminosidade.

A família Theridiidae foi a mais abundante no fragmento menor (PMM), o que pode sugerir que esta família seja mais tolerante à perda de habitat, como foi indicado por Miyashita, et al. (1998) que verificaram uma correlação negativa entre a abundância desta família e o tamanho dos fragmentos estudados em florestas de Tokyo e Yokohama, considerando que espécies desta família sejam menos sensíveis a tamanho do fragmento. A predominância da família Salticidae em ambientes de borda do PMP, sugere que estas famílias são mais tolerantes as alterações ambientais que são freqüentes em ambientes de vegetação mais aberta. Fato semelhante foi observado por Coyle (1981), que verificou que estas aranhas foram bastante abundantes em áreas desmatadas, Whitmore et al (2002), que encontraram um elevado número de Salticidae em ambiente de Savana na África, e Oliveira-Alves et al. (neste volume), que verificaram preferência de algumas espécies desta família por ambiente de borda. Este último estudo foi realizado no PMP.

Com relação aos indivíduos adultos, foram identificados 152, agrupados em 60 espécies e 19 famílias, sendo 37 espécies e 12 famílias no PMP e 30 espécies e 16 famílias no PMM (Tabela 1). Foram estimadas 46 espécies para PMP e 38 para PMM. No entanto, a curva do número estimado de espécies não alcançou a assíntota, indicando que as áreas apresentam riqueza ainda maior (Figura 2 e 3). As áreas não diferem quanto à riqueza estimada $(\mathrm{U}=65.000$; $\mathrm{p}=0$,2799) ou abundância de espécies ( $U=20755$, $p=0,1451$ ). Já a diversidade foi maior no PMP $(\mathrm{D}=8,1922)$ do que no PMM ( $D=6,8032)$. Estes resultados são considerados inusitados, já que fragmentos maiores geralmente possuem uma maior heterogeneidade de habitat e são capazes de comportar uma maior abundância e riqueza de espécies (Zimmerman \& Bierregaard 1986, Turner 1996, Rego et al. 2004). Porém, a semelhança encontrada nas áreas pode estar relacionada ao fato de que ambas as áreas sofrem processos semelhantes, como isolamento de área, perturbações e habitação no perímetro do parque. Tais eventos podem atuar de forma negativa na riqueza de espécies (van Dorb \& Opdan 1987, Viana \& Pinheiro 1998), principalmente no PMP.

Outro fato importante é o de que as coletas ocorreram na borda da mata, onde geralmente a vegetação nativa é alterada devido à perda de habitat, o que afeta o equilíbrio e a dinâmica das comunidades (Murcia 1995, Laurence et al. 1998). No entanto, isto parece não ocorrer no PMP pois Oliveira-Alves et al. (neste volume) encontraram na borda da mata uma elevada diversidade $\left(H^{\prime}=3,3741\right.$ - borda; $H^{\prime}=$ 3,0844 - centro). A semelhança encontrada na diversidade não era esperada, pois fragmentos menores que 10 ha, geralmente possuem maior vulnerabilidade, provocada por fatores ecológicos e ambientais (Azevedo et al. 2003, Sobrinho \& Schoereder 2003) que os tornam menos heterogêneos (Zimmerman \& Bierregaard 1986). Desta forma, o PMM deveria possuir uma diversidade inferior em relação ao PMP, já que a complexidade do ambiente influencia na capacidade de suportar um grande número de espécies (Rego et al. 2003). Porém, o fato do PMP ter o domínio de restinga arbórea e sofrer forte interferência antrópica pode estar influenciando negativamente sua diversidade já que, segundo Sá (2002), estes ecossistemas têm dificuldades de se regenerar após perturbações.

Desta forma as semelhanças encontradas entre os dois fragmentos de tamanhos tão distintos (PMM= nove ha e PMP= 425ha), sugere que a relação espécie-área, que parte do pressuposto de que quanto maior a área, maior será a riqueza e abundância em espécies, como foi observado por Schoereder et al. (2003), pode ter baixa influência na comunidade de aranhas que habitam fragmentos com histórico de perturbações antrópicas, possivelmente devido à substituição de espécies especialistas por espécies que tenham maior tolerância à variação ambiental, se adaptando a ambientes perturbados. Contudo, sugerimos que seja feita uma avaliação a posteriori de outros fatores, como a forma do fragmento, o tipo de vizinhança, efeito de borda, além de outras variáveis, i.e., composição florística, que possam interferir na dinâmica destes fragmentos e influenciar a comunidade destas aranhas.

Após as análises dos resultados obtidos, podem se estabelecer algumas hipóteses: (1) as semelhanças entre as áreas podem ser relativas a fatores históricos e ecológicos ligados à fragmentação, tais como isolamento de área, perturbações antrópicas e habitações no perímetro do parque, fatores estes que reduziriam a riqueza e diversidade de espécies, principalmente no PMP; (2) a dinâmica estrutural das áreas amostradas podem estar alterando a abundância, diversidade e riqueza de espécies.

\section{Agradecimentos}

Aos coordenadores do Centro ECOA, Moacir Tinôco e Anderson Abbehusen pela revisão dos manuscritos. Em especial para o Moacir Tinôco pela tradução do resumo. A Conder e a diretoria do Parque Municipal da Matinha, pelo apoio nos trabalhos de campo.

\section{Referências Bibliográficas}

AZEVEDO, A.R., SILVA, V.V. \& FERREIRA, A.M.M. 2003. Análise dos fragmentos florestais na Bacia o Rio Turvo, Médio Vale do Rio Paraíba do Sul, RJ. In: Anais de trabalhos completos do VI Congresso de Ecologia do Brasil. Fortaleza, p. 79-80. 
AYRES, M., AYRES, M.JR., AYRES, D.L., SANTOS, A.A. 2000. Bioestat 2.0. USP. São Paulo.

BURSLEM, D.R.R.P., GARWOOD, N.C. \& THOMAS, S.C. 2001. Tropical forest diversity-The plot thickens. Science 291: 606-607.

BRIGGS, J.C. 1996. Tropical diversity and conservation. Conservation Biology. 10:713-718.

CODDINGTON, J.A \& LEVI, H.W. 1991. Systematics and evolution of spider (Araneae). Annual Review of Ecology of Systematic 22:565-592.

COLLINGE, S.K. \& PALMER, T.M. 2002. The influences of patch shape and bounbary contrast on insect response to fragmentation in California grasslands. Ladscape Ecology. 17: 647-656.

COLWELL, R.K. 1997. EstimateS: Statistical Estimation of Species Richness and Shared Species from Samples. Version 5. User's Guide and application published at: http://viceroy.eeb.uconn.edu/estimates.

CONSERVATION INTERNATIONAL DO BRASIL, Fundação SOS Mata Atlântica, Fundação Biodiversitas, Instituto de Pesquisa Ecológica, Secretária de Meio Ambiente do Estado de São Paulo \& SEMAD/Instituto Estadual de Floresta-MG. 2000. Avaliação de ações prioritárias para a conservação da biodiversidade da Mata Atlântica e Campos Sulinos. Brasília MMA/SBF.

CONCEIÇÃO, A. de S., COSTA, J.A.S. \& FARIA, L.S.S. 1998. Plantas ruderais do entorno do campus da Universidade Católica do Salvador. In: Resumos do XLIX Congresso Nacional de Botânica. Universidade Federal da Bahia, Salvador, p.365.

CONROY, M.J. \& NOON, B.R. 1996. Mapping of species richness conservation of biological diversity: conceptual and methodological issues. Ecological Applications. 6: 763-773.

COYLE, F.A. 1981. Effects of clearcutting on the spiders community of a Southern Appalacian forest. Journal of Arachnology 9: 285 -298.

DÁRIO, R. F., ALMEIDA, A.F. 2000.Influência do corredor florestal sobre a Avifauna da Mata Atlântica. Scientia Forestalis 58: 99-109.

DESOUZA, O., SCHOEREDER, J.H., BROWN, V. \& BIERREGAARD, Jr. R.O. 2001. A Theoretical Overview of the Processes Determining Species Richness in Forest Fragments. In: Lessons from Amazônia - the ecology and conservation of a fragmented forest (Bierregaard, Jr. R.O, Gascon, C., Lovejoy, T.E. \& Mesquita, R., eds.). Sheridan Books, Michigan, p. 13-21.

ESPÍRITO-SANTO, F.D.B, OLIVEIRA-FILHO, A.T. (de), MACHADO, E.L.M, SOUZA, J.S., FONTES, M.A.M.L. \& MARQUES, J.J.G. de S. 2002. Variáveis ambientais e a distribuição de espécies arbóreas em um remanescente de Floresta Estacional Semidecídua Montana no Campos da Universidade Federal de Lavras, MG. Acta bot. bras. 16(3): 331-356.
FOELIX, R. F. 1996. Biology of Spiders. 2 ed. Oxford University Press, Oxford.

HARRIS, L.D. 1984.The fragmented forest: island biogeography theory and the preservation of biotic diversity. University of Chicago. Chicago, p.229.

HENDERSON, P.A. \& SEABY, R.M.H. 1998. Diversity 2.2. Disponível em: http//: www.irchouse.demon.co.uk/.

KAPOS, V. 1989. Effects of isolation on the water status of forest patches in the Brazilian Amazon. Journal of Tropical Ecology 5(2): 173-185.

KELT, D.A. 2000. Small mammal communities in rain forest fragments in Central Southern Chile. Biological Conservation 92: 345-358.

KREMEN, C., R.K.COLWELL, T.L.ERWIN, D.D.MURPHY, R.F.NOSS \& M.A. SANJAYAN. 1993. Terrestrial arthropod assemblages: their use In Conservation Planning. Conservation Biology 7: 796-808.

LAMBECK, R.J. 1997. Focal species: a multi-species umbrella for nature conservation. Conservation Biology 11:849-856.

LAURENCE, W.F., FERREIRA, L.V., RANKIN-DEMERONA, J.M. \& LAURENCE, S.G. 1998. Rain Forest fragmentation and dynamics of Amazonian tree communities. Ecology 79(6): 2032-2040.

LAWTON, J.H, BIGNELL, D.E., BOLTON, B., BLOEMERS, G.F., EGGLETON, P., HAMMOND, P.M., HODDA, M., HOLT, R.D., LARSEN, T.B., MAWDSLEY, N.A., STORK, N.E., SRIVASTAVA, D.S. \& WATT, A.D. 1998. Biodiversity inventories, indicator taxa and effects of habitat modification in tropical forest. Nature 391: 72-76.

MANTOVANI, W. 2003. Delimitação do bioma Mata Atlântica: implicações legais e conservacionistas. Ecossistemas Brasileiros: Manejo e Conservação. $1^{\circ}$ ed. Expressão Gráfica e Editora, Fortaleza, p.287-295.

MacARTHUR, R.H., WHITMORE, R.C. 1979. Passerine community composition and diversity in man-altered environments. Morgantown 7: 1-12.

MAY, R.M. 1988. How many species are there on earth? Science 241: 1441-1449.

METZGER, J.P. 2000. Tree functional group richness and spatial structure in a tropical fragmented landscape (SE Brazil). Ecological Applications 10: 1147-1161.

METZGER, J.P. 2003. Estratégias de Conservação baseadas em múltiplas espécies guarda-chuva: uma análise crítica. Ecossistemas Brasileiros: Manejo e Conservação. $1^{\circ}$ ed. Expressão Gráfica e Editora, Fortaleza.

MIYASHITA, T., Shinkai, A., Chida, T. 1998. The effects of forest fragmentation on web spider communities in urban areas. Biological Conservation 86: 357-364. 
MURCIA, C. 1995. Edge effects in fragmented forests: implications for conservation. Trends in Ecology and Evolution 10: 58-62.

MYERS, N. 1997. Florestas tropicais e suas espécies-sumindo, sumindo...? In: Biodiversidade(Wilson, E.O. \& F.M. Peter, eds.). Ed. Nova Fronteira, Rio de Janeiro, p. 36-45.

MYERS, N., MITTERMEIER, R.A., MITTERMEIER, C.G., FONSECA, G.A.B. \& KENT J. 2000. Biodiversity hotspots for conservation priorities. Nature 403: 853-858.

OLIVEIRA-ALVES, A. PERES, M.C.L, DIAS, M.A., CAZAISFERREIRA, G. S. Estudo das comunidades de aranhas (Arachnida: Araneae) em ambiente de Mata Atlântica no Parque Metropolitano de Pituaçu - PMP, Salvador, Bahia. Biotaneotropica (submetido).

PEARSON, D.L. 1994. Selecting indicator taxa for the quantitative assessment of biodiversity. The Royal Society 345: 75-79.

PERES, M.C.L. 2001. Estudo das comunidades de aranhas (Araneae: Arachnida) em clareiras naturais e floresta madura de Floresta Atlântica - Parque Estadual de Dois Irmãos (Recife - Pernambuco - Brasil). Dissertação de Mestrado. UFPE, Recife.

PLATNICK. 1995. An abundance of spiders. Natural Histology, p. 52-52.

PLATNICK, N.I. 2004. The World Spider Catalog, version 5.0 (online) - Disponível em: http:/research.amnh.org/ entomology/spiders/catalog 81-87/COUNTS.htm. Acesso: 10/10/2004.

PRIMACK, R.B. \& RODRIGUES, E. 2002. Biologia da Conservação. 3 ed. Vida. Londrina.

REGO, F.N.A.A., VENTICINQUE, E.M. \& BRESCOVIT,A.D. 2003. Fragmentos florestais reduzem a abundância da comunidade de aranhas do sub-bosque, na Amazônia Central: Considerações sobre o estudo e a conservação de áreas degradadas. In: Anais do IV Congresso de Ecologia do Brasil, p. 237.

RUSHTON, S.P. TOPPING, C.J. \& EYRE, M.D. 1987. The habitat preferences of grassland spiders as identified using detrended correspondence analysis (Decorama). Bull. of Britich Arachnological Society 7: 165-170.

SÁ, C.F.C (de). 2002. Regeneração de um trecho de floresta de restinga na Reserva Ecológica Estadual de Jacarepiá, Saquarema, Estado do Rio de Janeiro: II - Estrato arbustivo. Rodriguésia 53 (82): 5-23.

SANTOS, A.J. 1999. Diversidade e composição em espécies de aranhas da Reserva Florestal da Companhia Vale do Rio Doce (LINHARES/ES). Dissertação de Mestrado. UNICAMP. Campinas.
SANTOS, A.J. 2002. Análise de riqueza em espécies em inventários de biodiversidade: problemas e possíveis soluções. In: Anais de Resumos do $3^{\circ}$ Encuentro de Aracnólogos Del Cono Sur. Córoba, p.110.

SCHOEREDER, J.H., SPERBER, C.F., SOBRINHO, T.G., RIBAS, C.R., GALBIATI, C., MADUREIRA, M.S. 2003. Por que a riqueza de espécies de insetos é menor em fragmentos menores? Processos locais e regionais. Ecossistemas Brasileiros: Manejo e Conservação. $1^{\circ}$ ed. Expressão Gráfica e Editora, Fortaleza, p.31-38.

SIMÓ, M., PÉREZ-MILES F., PONCE DE LEÓN, A.F.E., MENEGHEL M. 1994. Relevamiento de Fauna de la quebrada de los cuervos; area natural protegida. Bol. Soc. Zool. Del Uruguay 2: 1-20.

SOBRINHO, T.G. \& SCHOEREDER, J.H. 2003. Fragmentação florestal afeta número e composição de espécies de formigas (Hymenoptera: Formicidae)? In: Anais de trabalhos completos do VI Congresso de Ecologia do Brasil. Fortaleza, p. 144-145.

STEFFAN-DEWENTER, I. 2003. Importance of habitat area and latscape context for species richness of bees and wasps in fragmented orchard meadows. Conservation Biology 17: 1036-1044.

TOTI, D.S., F.A. COYLE \& J.A. MILLER. 2000. A structured inventory of appalachian grass bald and heath bald spider assemblages and a test of species richness estimator performance. Journal of Arachnology 28: 329-345.

TURNER, I.M. 1996. Species loss in fragments of tropical rain forest: a review of the evidence. Journal of Applied Ecology 33: 200-209.

VAN DORB, D. \& OPDAN, P.F.M. 1987. Effects of patch size, isolation and regional abundance on forest bird communities. Landscape Ecology 1: 59-73.

VIANA, V.M. 1995. Conservação da biodiversidade de fragmentos de florestas tropicais em paisagens intensamente cutivadas. In: Abordagens interdisciplinares para a conservação da biodiversidade e dinâmica do uso da terra no novo mundo. Gainesville: Conservation International do Brasil/Universidade Federal de Minas Gerais/University of Florida, p. 135-154.

VIANA, V.M \& PINHEIRO, L.A.F.V. 1998. Conservação da biodiversidade em fragmentos florestais. Série Técnica IPEF 12(32): 25-42.

VIEIRA, M.V. 2003. A dinâmica temporal e espacial de populações e comunidades animais da Floresta Pluvial Atlântica: pequenos mamíferos como estudo de caso. Ecossistemas Brasileiros: Manejo e Conservação. $1^{\circ}$ ed. Expressão Gráfica e Editora, Fortaleza. 
ZIMMERMAN, B.L. \& BIERREGAARD, R.O. Jr.1986. Relevance of the equilibrium theory of island biogeography with an example from Amazonia. J. Biogeography 13: 133-143.

WISE, D.H. 1993. Spiders in ecological webs. Cambridge University Press, Cambridge.

WHITMORE, C., SLOTOW, R., CROUCH, T.E. \& DIPPENAAR-SCHOEMAN, A.S. 2002. Diversity of spiders (Araneae) in a Savanna Reserve, Northern province, south Africa. The Journal of Arachnology 30: 344-356.

Título: Aspectos comparativos das comunidades de aranhas (Araneae) em dois remanescentes de Mata Atlântica da Bahia, Brasil.

Autores: Kátia Regina Benati, João Pedro Souza-Alves, Elizabete Alves Silva, Marcelo Cesar Lima Peres e Érica Oliveira Coutinho

Biota Neotropica, Vol. 5( número 1a): 2005

http://www.biotaneotropica.org.br/v5n1a/pt/ abstract?article+BN005051a2005

Recebido em 07/12/2003 - Aceito em 01/02/2005

ISSN 1676-0603

http://www.biotaneotropica.org.br 